\title{
Exosomes secreted by stem cells from human exfoliated deciduous teeth contribute to functional recovery after traumatic brain injury by shifting microglia M1/M2 polarization in rats
}

Ye Li ${ }^{1,2}$, Yuan-Yuan Yang ${ }^{2}$, Jia-Li Ren², Feng Xu ${ }^{3,4}$, Fa-Ming Chen ${ }^{5}$ and Ang $\mathrm{Li}^{1,2^{*}}$

\begin{abstract}
Background: Traumatic brain injury (TBI) is one of the major causes of mortality and disability for all ages worldwide. Mesenchymal stem cells (MSCs)-originated exosomes have provided therapeutic effects. However, as an indispensable component of MSCs, whether odontogenic stem cell-generated exosomes could benefit TBI is still unclear. Thus we aimed to explore the potential of stem cells from human exfoliated deciduous teeth-originated exosomes (SHED-Ex) for the management of TBI.
\end{abstract}

Methods: First, a transwell system was used to co-culture activated BV-2 microglia cells with SHED. The secretion levels of neuroinflammatory factors and nitrite were evaluated by enzyme-linked immunosorbent assay (ELISA) and Griess assay. Furthermore, purified SHED-Ex were co-cultured with activated BV-2. ELISA, Griess assay, flow cytometry, immunofluorescence, and qRT-PCR were performed to test the levels of inflammatory factors as well as the microglia phenotype. Finally, SHED and SHED-Ex were locally injected into TBI rat models. Basso, Beattie, and Bresnahan (BBB) scores were chosen to evaluate the motor functional recovery. Histopathology and immunofluorescence were performed to measure the lesion volume and neuroinflammation.

Results: As a result, SHED-Ex could reduce neuroinflammation by shifting microglia polarization. The administration of SHED-Ex improves rat motor functional recovery and reduces cortical lesion compared with the control group 2 weeks post-injury $(P<0.05)$.

Conclusions: The current study demonstrates for the first time that SHED-Ex contribute a therapeutic benefit to TBI in rats, at least in part by shifting microglia polarization to reduce neuroinflammation. The use of odontogenic stem cells, and indeed their exosomes, may be expanded for the treatment of TBI or other neurological disorders.

Keywords: Traumatic brain injury, Stem cells from human exfoliated deciduous teeth, Exosomes, Microglia, Neuroinflammation

\footnotetext{
* Correspondence: drliang@mail.xjtu.edu.cn

${ }^{1}$ Key Laboratory of Shaanxi Province for Craniofacial Precision Medicine

Research, Xi'an, Shaanxi, China

${ }^{2}$ Department of Periodontology, College of Stomatology, Xi'an Jiaotong

University, Xi Wu Road No.98, Xi'an, Shaanxi 710004, China

Full list of author information is available at the end of the article
} 


\section{Background}

Stem cells from human exfoliated deciduous teeth (SHED) have been reported as an attractive source for tissue engineering and stem cell transplantation $[1,2]$. With the representative characteristics of mesenchymal stem cells (MSCs), SHED display several advantages in the application of regenerative medicine. SHED are derived from dental pulps from young patients and hence represent a more immature population of postnatal stem cells with a higher proliferation rate [3]. Besides, SHED are more accessible for clinical application for noninvasive retrieval compared with MSCs from bone marrow, cartilage, muscle, and adipose tissue [4]. In addition, human exfoliated deciduous teeth are "disposable" tissues that are often taken as medical waste [5]. Therefore, fewer ethical concerns exist. With these advantages, SHED are recommended as a promising source of cell types for tissue engineering and have been utilized for the successful regeneration of a number of dental tissues in animal models. Interestingly, these cells also have the potential to give rise to non-dental cell lineages for use in the treatment of many systematic diseases, such as systemic lupus erythematosus, photoaging, and type 1 diabetes [6-9].

Defined as damage to the brain caused by external mechanical force, traumatic brain injury (TBI) has long been a major cause of mortality worldwide [10]. At present, few effective strategies or therapeutic interventions have appeared for treating TBI. Holding promise in the management of TBI, stem cell therapy has been shown to be an effective way for functional improvement [11]. However, it remains unclear whether SHED could benefit TBI as well. TBI is always followed by a cascade of events mediated by microglia, one of the main active cells of the central nervous system [12]. At the beginning, pro-inflammatory effects predominate, and a large number of inflammatory factors were released. Then, there is a transition of the microglia phenotype. They transit from the M1 polarization state to M2 to suppress pro-inflammatory mediators as well as enhance tissue repair [13]. However, chronic effects often occur because of ineffective inhibition of the pro-inflammatory process. Therefore, shifting microglia from M1 to M2 could be a target for the treatment of TBI. Given that neural stem cells (NSCs) benefit TBI patients by alternating the microglial polarization, we investigated here whether SHED could modulate the host microglia in a manner similar to that of NSCs.

Recently, increasing evidence has demonstrated that small-membrane vesicle exosomes with a size of approximately $30-100 \mathrm{~nm}$ in diameter play a pivotal role in stem cell therapy [14]. What is more, administrations of exosomes have demonstrated a therapeutic effect in multiple injury models [15-17]. It is believed that exosomes hold great potential as cell-free therapies because of their safety and low immunogenicity $[18,19]$. Therefore, we hypothesize that exosomes derived from SHED (SHED-Ex) could be beneficial for TBI and thus provide a better way for treatments. Therefore, the present study primarily investigated whether SHED-Ex could benefit microglia polarization transition in vitro. More importantly, an experimental injury model was established with locally administered SHED-Ex to investigate motor functional recovery.

\section{Methods \\ Culture of BV-2 microglia and TERT-SHED}

Two immortalized cell lines were used in the present study. BV-2 was obtained from The Chinese Academy of Sciences, and TERT-immortalized SHED were resuscitated from TERT-SHED in our previous report [20]. Cells were conventionally cultivated with Dulbecco's modified Eagle's medium (DMEM, Gibco, Grand Island, NE, USA) containing 1\% penicillin/streptomycin (Gibco) and $10 \%$ fetal bovine serum (FBS, Gibco) at $37^{\circ} \mathrm{C}$.

\section{Characterization of BV-2 microglia and TERT-SHED}

The microglia phenotype of BV-2 was characterized by flow cytometry and immunofluorescence. Cells were stained with FITC-labeled CD11b and APC-labeled F4/ 80 antibodies and analyzed by BD FACSCalibur flow cytometer (BD Biosciences, San Jose, CA, USA). Rat antimouse CD11b, Iba-1, and F4/80 were used in the immunofluorescence, and the protocol was as previously described [21]. The results were analyzed by Olympus FSX100 fluorescence microscope (Olympus Corp., Tokyo, Japan). The characterization of TERT-SHED was performed previously [20].

\section{Microglia stimulation and ELISA}

BV-2 were incubated with $1 \mu \mathrm{g} / \mathrm{ml}$ lipopolysaccharide (LPS) for $24 \mathrm{~h}$ to induce a pro-inflammatory phenotype. Enzyme-linked immunosorbent assay (ELISA) was performed to measure the concentrations of tumor necrosis factor alpha (TNF- $\alpha$ ) and interleukin-6 (IL-6) in cell supernatants. The experiments were all performed in triplicate three times.

\section{Transwell assay}

The co-culture experiment was performed by transwell assay as shown in Fig. 2a, the exchange of factors was allowed without cell-to-cell contact. In a transwell system, activated BV-2 were plated in the lower chamber, and TERT-SHED were plated in the upper chamber. Monocultures of activated BV-2 microglial cells were processed as controls. 


\section{Exosome enrichment and characterization} TERT-SHED were cultured in DMEM with exosomefree FBS (SBI, Palo Alto, CA, USA) for $48 \mathrm{~h}$. The cell culture media was then collected and mixed with the ExoQuick exosome isolation solution, and the following steps were performed in line with the manufacturer's instructions. PE-labeled CD81, CD63, and CD9 were used to evaluate the characteristics of the isolated exosomes by flow cytometry. For further validation of SHED-Exo, $10 \mu \mathrm{M}$ GW4869 (Sigma-Aldrich, St Louis, MO,, USA) was used to inhibit exosomes release from SHED. Equal amounts of protein were extracted from SHED-Exo, SHED, and exosomes from GW4869treated SHED for western blotting to detect the expression of CD9 and CD63.

\section{Electron microscopy}

The enriched exosomes were first loaded onto a carboncoated grid, and remained for $7 \mathrm{~min}$. With the excess fluid removed, samples were then stained in 3\% phosphotungstic acid ( $\mathrm{pH}$ 6.9) for $5 \mathrm{~min}$. Transmission electron microscopy (TEM) was used for the analysis.

\section{Exosome labeling and internalization}

Purified exosomes were labeled with CM-DiI (red) as previously described [22]. BV-2 microglia were seeded in 24-well plates for overnight attachment. The labeled exosomes were added the next day for a $48 \mathrm{~h}$ incubation. The nuclei were stained with DAPI before image acquisition.

\section{Griess assay}

As the supernatant accumulation of nitrite is an indicator of the production of nitric oxide (NO), Griess assay was performed to determine the nitrite concentration. BV-2 microglia cells were activated as previously described, and different concentrations with equal volume (50, 100, 200, $400 \mu \mathrm{g} / \mathrm{ml}$ ) of SHED exosomes were added into the culture medium. We incubated $50 \mu \mathrm{l}$ of each supernatant with $50 \mu \mathrm{l}$ Griess reagent (Promega, Madison, WI, USA) for $8 \mathrm{~min}$. The absorbance was then measured at $520 \mathrm{~nm}$. With a standard curve as the reference, the nitrite concentration was calculated.

\section{Flow cytometry and immunofluorescence}

BV-2 microglia cells were activated as previously described, and different concentrations (50, 100, 200, $400 \mu \mathrm{g} / \mathrm{ml}$ ) of SHED exosomes were added into the culture medium. Rat anti-mouse CD11b and CD68 were used to evaluate the M1 phenotype of the microglia. The changes were assessed by flowjo3.4.1 (FlowJo, LLC, Ashland, OR, USA). For immunofluorescence, confocal microscopy was used to analyze the images.

\section{qRT-PCR}

Total RNA was extracted, cDNA was synthetized and real-time PCR was performed. Primers used are shown in Table 1. GAPDH was used as the reference. ABI 7500 Sequence Detection System (Applied Biosystems, Foster City, CA, USA) was used to analyze the results).

Table 1 Primer sequences

\begin{tabular}{|c|c|c|c|c|}
\hline & Gene & Gene bank accession & Primer sequence $\left(5^{\prime}-3^{\prime}\right)$ forward primer reverse primer & Product (bp) \\
\hline \multirow[t]{2}{*}{ Reference } & $\beta$-actin & NM_007393 & GCCCTGAGGCTCTITTCCAG & 51 \\
\hline & & & TGCCACAGGATTCCATACCC & \\
\hline \multirow[t]{10}{*}{ M1 phenotype } & CD11b & NM_001082960.1 & GAGCAGCACTGAGATCCTGTTTAA & 64 \\
\hline & & & ATACGACTCCTGCCCTGGAA & \\
\hline & CD86 & NM_019388.3 & ACGATGGACCCCAGATGCACCA & 88 \\
\hline & & & GCGTCTCCACGGAAACAGCA & \\
\hline & CD16 & XM_006496658.2 & TGTGTGTCGTCGTAGACGGT & 396 \\
\hline & & & TTCGCACATCAGTGTCACCA & \\
\hline & $\mathrm{MHCll}$ & NM_010382.2 & ACAGGAATTGTGTCCACGGG & 472 \\
\hline & & & AAGGCCTGGGTCAGGGATAA & \\
\hline & iNOS & NM_010927.3 & GACGAGACGGATAGGCAGAG & 80 \\
\hline & & & GTGGGGTTGTTGCTGAACTT & \\
\hline \multirow[t]{6}{*}{ M2 phenotype } & CD206 & NM_000237.2 & TCAGCTATTGGACGCGAGGCA & 105 \\
\hline & & & TCCGGGTTGCAAGTTGCCGT & \\
\hline & $\mathbb{L L}-10$ & NM_010548.2 & CGGCTGAGGCGCTGT & 51 \\
\hline & & & TGCCTTGCTCTTATTTTCACAGG & \\
\hline & $\operatorname{Arg}-1$ & NM_007482.3 & AGCCAATGAAGAGCTGGCTGGT & 91 \\
\hline & & & AACTGCCAGACTGTGGTCTCCA & \\
\hline
\end{tabular}




\section{Animal model}

A well-established rat TBI model was utilized in the present study via the "free-falling method" [23]. Adult male Wistar rats weighing 220-240 g were first anesthetized with chloral hydrate intraperitoneally. A stereotactic frame was used to induce the injury. A $2 \mathrm{~mm}$ diameter circular window was drilled $1 \mathrm{~mm}$ adjacent to the left side of anterior fontanelle, which corresponded to the cortical motor functional area. A $20 \mathrm{~g}$ weight was used to hit the injured area. The craniotomy was then closed. The impact of this injury is nearly equal to a moderate TBI in humans.

\section{Assessment of neurological function}

Basso, Beattie, and Bresnahan (BBB) scores were used to assess the neurological motor function before TBI and on the 1st, 3rd, 7th, 14th and 21st day after TBI. The assessment was performed by three investigators blinded to the experimental groups [24]. BBB is a complex behavioral test with a scoring range of $0-21$ that evaluates long-term functional recovery. The stages are divided into the early phase, intermediate phase, and late phase of recovery (score from 0-7, 8-13, 14-21 respectively).

\section{Tissue preparation, H\&E staining, and immunofluorescent} staining

After anesthesia, the brains were extracted and fixed by $4 \%$ paraformaldehyde, and then tissues were dehydrated in the $15 \%$ sucrose solution followed by a $30 \%$ sucrose solution. OCT-embedded tissues are ready for staining. $H \& E$ staining of the tissues was performed as previously reported [25]. Immunostaining was performed to identify CD68-positive cells in the lesion zone.

\section{Statistical analysis}

Student's $t$ test and one-way ANOVA were used for the evaluation of the differences among groups. A $P$ value less than 0.05 was considered as the significant difference.

\section{Results}

BV-2 cell line displays a normal microglia phenotype

To characterize the phenotype of the BV-2 cell line, we first evaluated the expression levels of classical markers that are used to identify microglia: CD11b, Iba-1, and F4/80. First, microglia surface markers CD11b and F4/ 80 were analyzed by flow cytometry. Both the markers detected high expression levels (Fig. 1a); isotype controls accounting for nonspecific binding are shown in gray. The expression of CD11b, Iba-1, and F4/80 were further confirmed by fluorescence microscopy (Fig. 1b); all three markers were strongly detected in BV-2 cells. These combined data suggest that BV-2 cells formed a pure population with biomarkers associated with normal microglia.

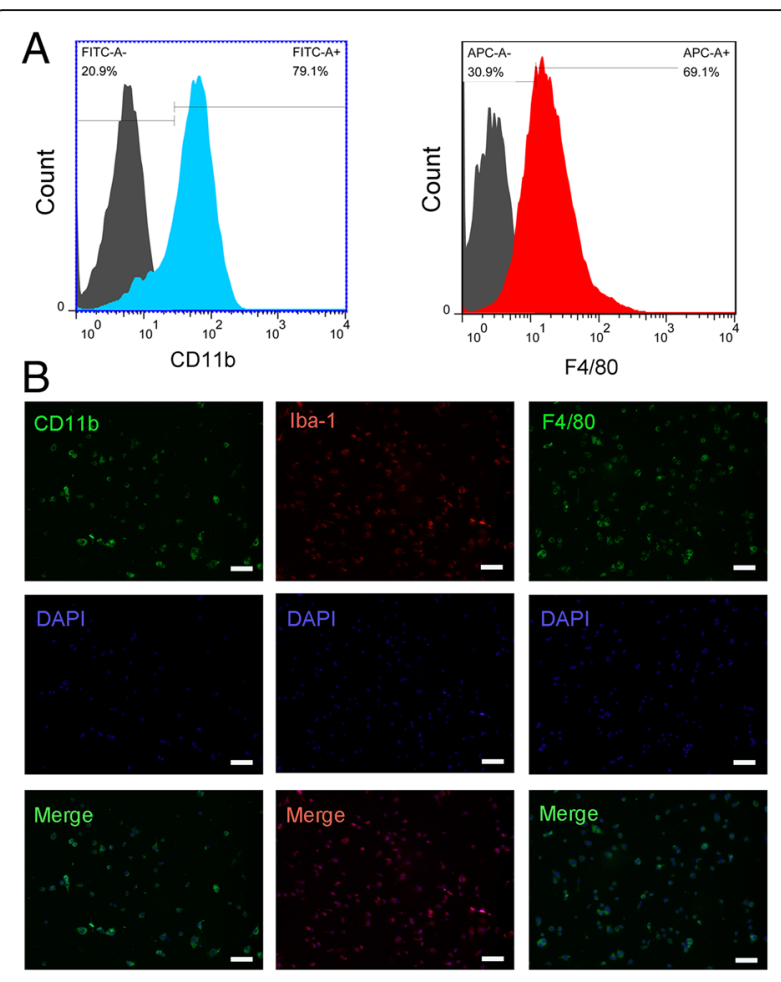

Fig. 1 Phenotyping of BV-2 microglia. a Flow cytometry histograms showed the expression levels of surface markers: CD11b (FITC, blue peaks) and F4/80 (APC, red peaks); the numbers of positive cells are indicated at the top right corner. $\mathbf{b}$ Representative images of BV-2 microglia with respect to immunofluorescence observation showing positive expression of CD11b, Iba-1, and F4/80. Scale bar $=100 \mu \mathrm{m}$

The immunomodulatory effect on SHED co-cultured microglia

A transwell co-culturing system was used to evaluate the effect of SHED on BV-2 microglia (Fig. 2a). A Griess assay and ELISA test were used to determine the levels of nitrite and of inflammatory factors TNF- $\alpha$ and IL-6 that are secreted by microglia. Nitrite secreted by activated microglia after co-culturing for $48 \mathrm{~h}$ were greatly decreased compared with the single activated microglia group (Fig. 2b). TNF- $\alpha$ and IL-6 showed a similar tendency to decrease (Fig. 2c, d).

\section{Identification of SHED-Ex}

When the purified exosomes generated from SHED were observed by TEM, most of the exosomes had a characteristic morphology with a size ranging from 30 to $100 \mathrm{~nm}$ (Fig. 3a). Then the expression of surface exosomal markers CD9 and CD63 were confirmed by western blotting (Fig. 3b). Flow cytometry showed that surface exosomal markers such as CD9, CD63, and CD81 were highly expressed (Fig. 3c). Moreover, the internalization of the exosomes was tested. The exosomes were first labeled with CM-DiI and were then added into the BV-2 microglia culture system for incubation. After $48 \mathrm{~h}$ we 

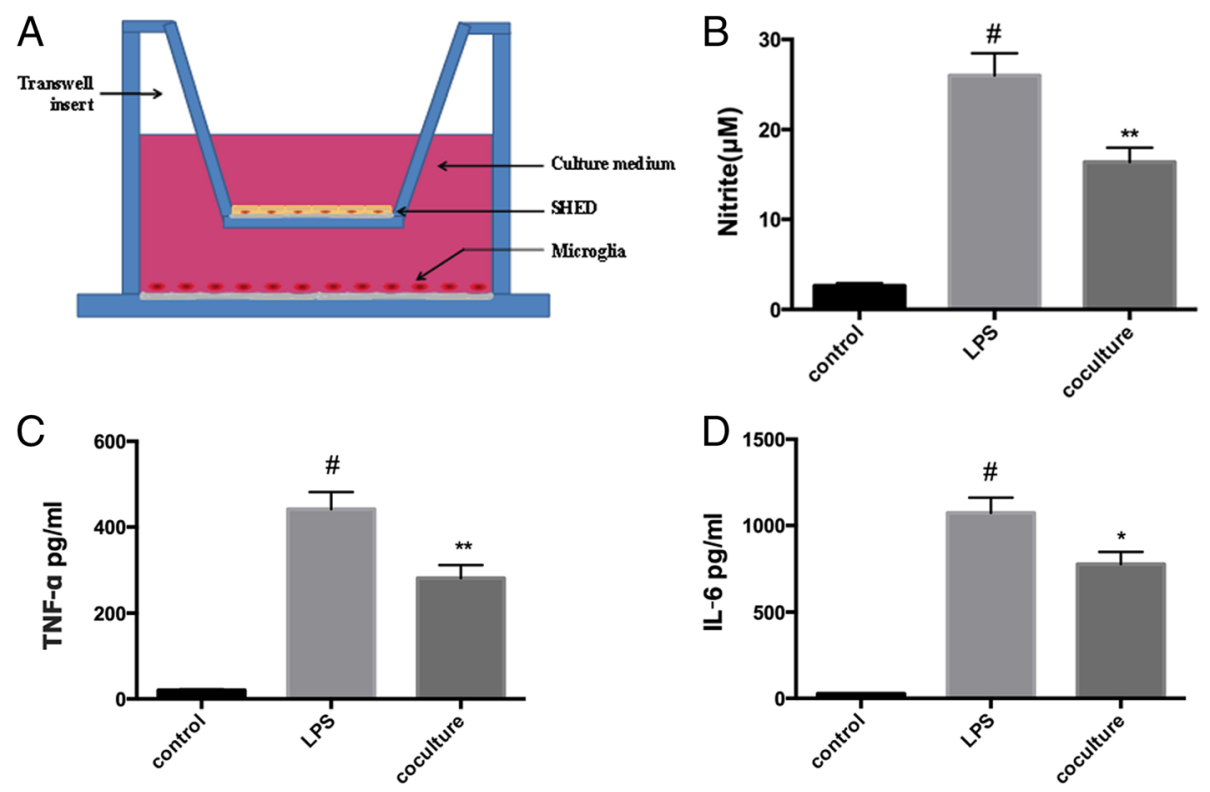

Fig. 2 The immunomodulatory effect of SHED on BV-2 microglia. a The co-culture system of SHED and BV-2 microglia. In a transwell system, SHED and BV-2 microglia were cultured together, as shown. $\mathbf{b}$ Inhibitory effect of SHED on the production of nitrite. BV-2 were treated with 1 $\mu \mathrm{g} / \mathrm{ml}$ LPS for $24 \mathrm{~h}$ and then followed by SHED co-culture. The content of nitrite was measured by Griess assay. $\mathbf{c}$, d The effect of SHED on proinflammatory cytokines TNF-a and IL-6. An ELISA assay was performed. ${ }^{\#} P<0.05$ compared with nonactivated microglia cells. ${ }^{*} P<0.05$, ${ }^{* * *} P<0.01$ compared with LPS-only treated cells. IL-6 interleukin-6, LPS lipopolysaccharide, SHED stem cells from human exfoliated deciduous teeth, TNF-a tumor necrosis factor alpha

could detect strong red fluorescence in the microglia cells (Fig. 3d) and the percentage of DiI cells increased along with the incubation time (Fig. 3e).

\section{SHED-Ex display a robust response to pro-inflammatory stimuli}

To determine the effects of SHED-Ex on microglia, a Griess assay, an ELISA test, flow cytometry and immunofluorescence were used. Activated microglia were treated with different concentrations of SHED-Ex (50, 100, 200, $400 \mu \mathrm{g} / \mathrm{ml}$ ) for $48 \mathrm{~h}$. We sought to determine whether SHED-Ex could affect microglia in response to the proinflammatory process. The results of the Griess assay demonstrated that SHED-Ex decreased the nitrite concentration in a dose-dependent manner (Fig. 4a). What is more, an ELISA test was used to measure the concentrations of TNF- $\alpha$ and IL- 6 in the microglia supernatants. Both the cytokines were significantly decreased in a dosedependent manner in the SHED-Ex group compared with the single LPS group (Fig. 4b). The expression levels of CD11b and CD68 were next evaluated to measure the pro-inflammatory phenotype. The flow cytometry results showed that SHED-Ex strongly decreased the expression level of CD68 in a dose-dependent manner, while the influence in CD11b is not obvious (Fig. 4c). In accordance with the flow cytometry results, the immunofluorescence results demonstrated that SHED-Ex significantly affected the expression level of CD68 (Fig. 4d). The combined results suggest that SHED-Ex could display a robust response to pro-inflammatory stimuli in a dose-dependent manner.

\section{Microglial M1 phenotype polarization was prevented by SHED-Ex while M2 phenotype was promoted}

Microglia M1/M2-associated markers were detected via mRNA levels. The data showed that the mRNA levels of M1 polarization-associated markers CD11b, CD86, CD16, MHCII, and iNOS were all significantly decreased in BV-2 microglia (Fig. 5a). While the mRNA levels of M2 polarization-associated markers CD206, IL-10 and ARGINASE 1 were all significantly increased in BV-2 microglia (Fig. 5b) compared with single LPS treatment.

\section{Administration of SHED-Ex significantly promotes functional motor recovery in rats after TBI}

Functional motor measurement was performed in rats using standardized BBB scores. The recovery of motor function is graded on a scale of 0-21; the higher the score, the better the recovery. The BBB score was 10-12 in rats with TBI (all the groups) on day 1 post-TBI, indicating that motor deficits in all rats were comparable at the beginning. A significant promotion was found over time in the SHED-treated and SHED-Ex-treated animals from day 3-21 compared with day 1 post-injury, suggesting that there is a significant spontaneous recovery in treatment groups after TBI. More importantly, compared with the 
A

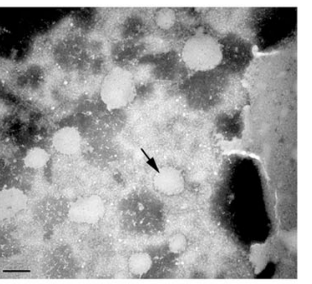

C
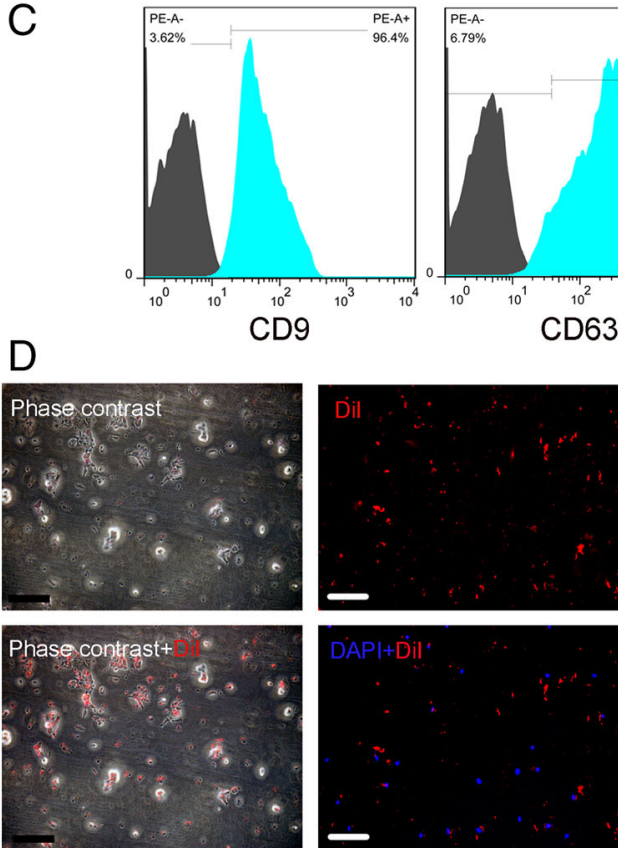

B
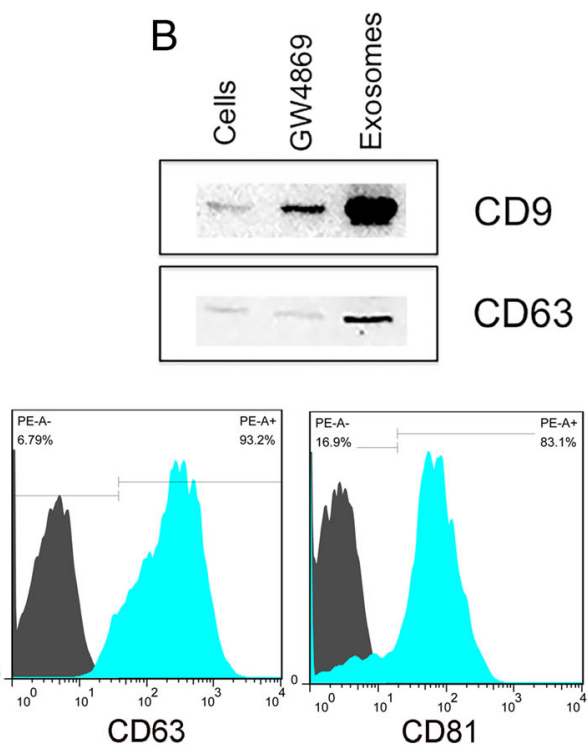

$E$

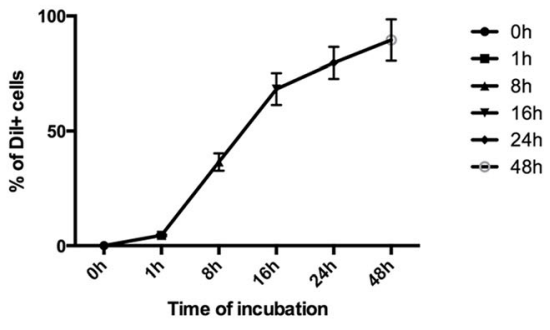

Fig. 3 Characterization of SHED-Ex and internalization assay. a Morphologic observation of SHED-Ex by transmission electron microscopy (scale bar $=50 \mathrm{~nm})$. b Surface exosomal markers (CD9, CD63) were analyzed using Western blotting in SHED, exosomes from GW4869-treated SHED, and SHED-Exo. c Flow cytometric analysis showing the presence of CD9, CD63, and CD81 in exosomes derived from SHED. d Immunofluorescence image showing the internalization of CM-Dil-labeled exosomes (red) by microglia (scale bar $=150 \mu \mathrm{m}$ ). e Dil uptake was analyzed by flow cytometry with different incubation times

SHED group, functional motor recovery was significantly increased in the $1000 \mu \mathrm{g} / \mathrm{ml}$ SHED-Ex group (Fig. 6b). The cortical lesion volume was measured in the different groups at $48 \mathrm{~h}$ and 2 weeks post-TBI (Fig. 6c). Significant recovery was observed between the single TBI group and the $1000 \mu \mathrm{g} / \mathrm{ml}$ SHED-Ex group. Microglia were identified with CD68 immunofluorescence staining in the brain after TBI. As shown in Fig. 6d, the single TBI group demonstrated an increased density of CD68+ cells compared with the sham group. SHED-Ex could reduce the CD68+ cell density. From the statistical data, SHED-Ex treatment significantly inhibits CD68+ cells in rat brain (Fig. 6e).

\section{Discussion}

TBI is one of the leading causes of severe disability and mortality for all ages worldwide. Affected patients are often accompanied by resultant motor or cognitive dysfunction, leaving devastating effects on the ability to continue with a normal life. As a potential treatment strategy, stem cell therapy has received much attention over the years. Neural stem cells are perhaps the fundamental choice for transplantation, but the availability is somehow limited, and there are not always enough cells for therapy $[26,27]$. Interestingly, mesenchymal stem cells from bone marrow, adipose tissue, and umbilical cords showed an attractive therapeutic effect by improving the impaired function in animal models [28-30]. However, as one of the important components of MSCs, it remains unclear whether odontogenic stem cells could benefit TBI as well. Until now, with mesenchymal stem cell characteristics, odontogenic stem cells that have been well characterized are as follows: dental follicle progenitor cells (DFPCs), dental pulp stem cells (DPSCs), stem cells from apical papilla (SCAP), periodontal ligament stem cells (PDLSCs), 


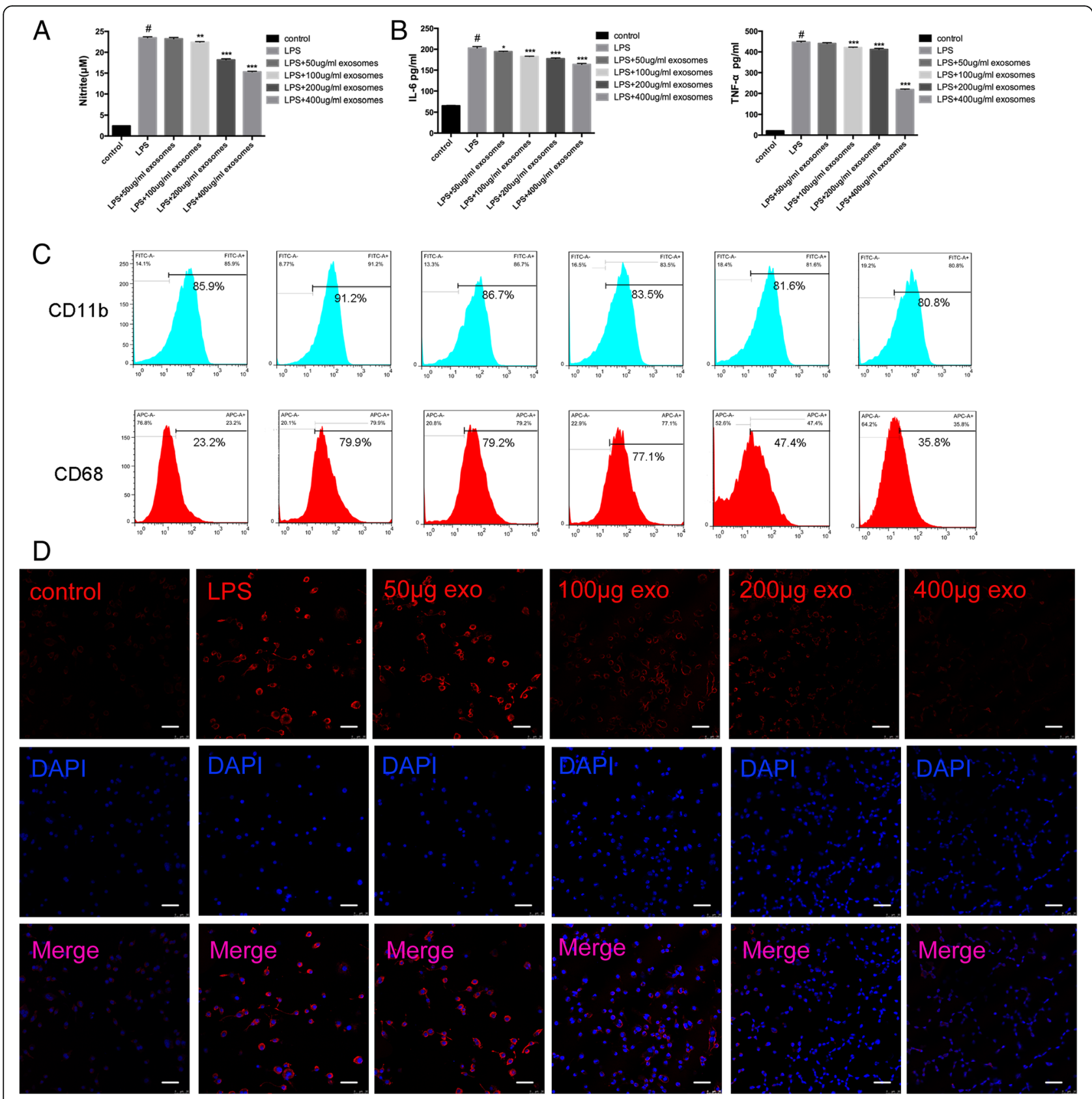

Fig. 4 SHED-Ex displays a robust response to pro-inflammatory stimuli. a Griess assay for the microglia production of nitrite with different concentrations of SHED-Ex. b ELISA assay for pro-inflammatory cytokines TNF-a and IL-6 with different concentrations of SHED-EX. c Flow cytometry for the expression levels of CD11b and CD68 of microglia with different concentrations of SHED-Ex. d Immunofluorescence staining and confocal analysis for CD68. ${ }^{\#} P<0.05$ compared with non-activated microglia cells. ${ }^{*} P<0.05,{ }^{* *} P<0.01,{ }^{* * *} P<0.001$ compared with LPS-only treated cells. IL-6 interleukin-6, LPS lipopolysaccharide, TNF-a tumor necrosis factor alpha

and stem cells from exfoliated deciduous teeth (SHED) [31]. Among them, SHED strongly express NANOG, SOX2 , and OCT-4, all of which are embryonic stem cell markers, and have been reported to exhibit outstanding craniomaxillofacial tissue regeneration capacities [32]. The current study was designed as a first attempt to use SHED for the administration in TBI.
In our present in vitro study, the concentration of inflammatory factors TNF- $\alpha$ and IL- 6 was evaluated with or without SHED treatment. It was found that SHED could significantly reduce the secretion of inflammatory factors by activated microglia. Previously, the administration of bone marrow mesenchymal stem cells in a TBI rat model also demonstrated the anti-inflammatory 

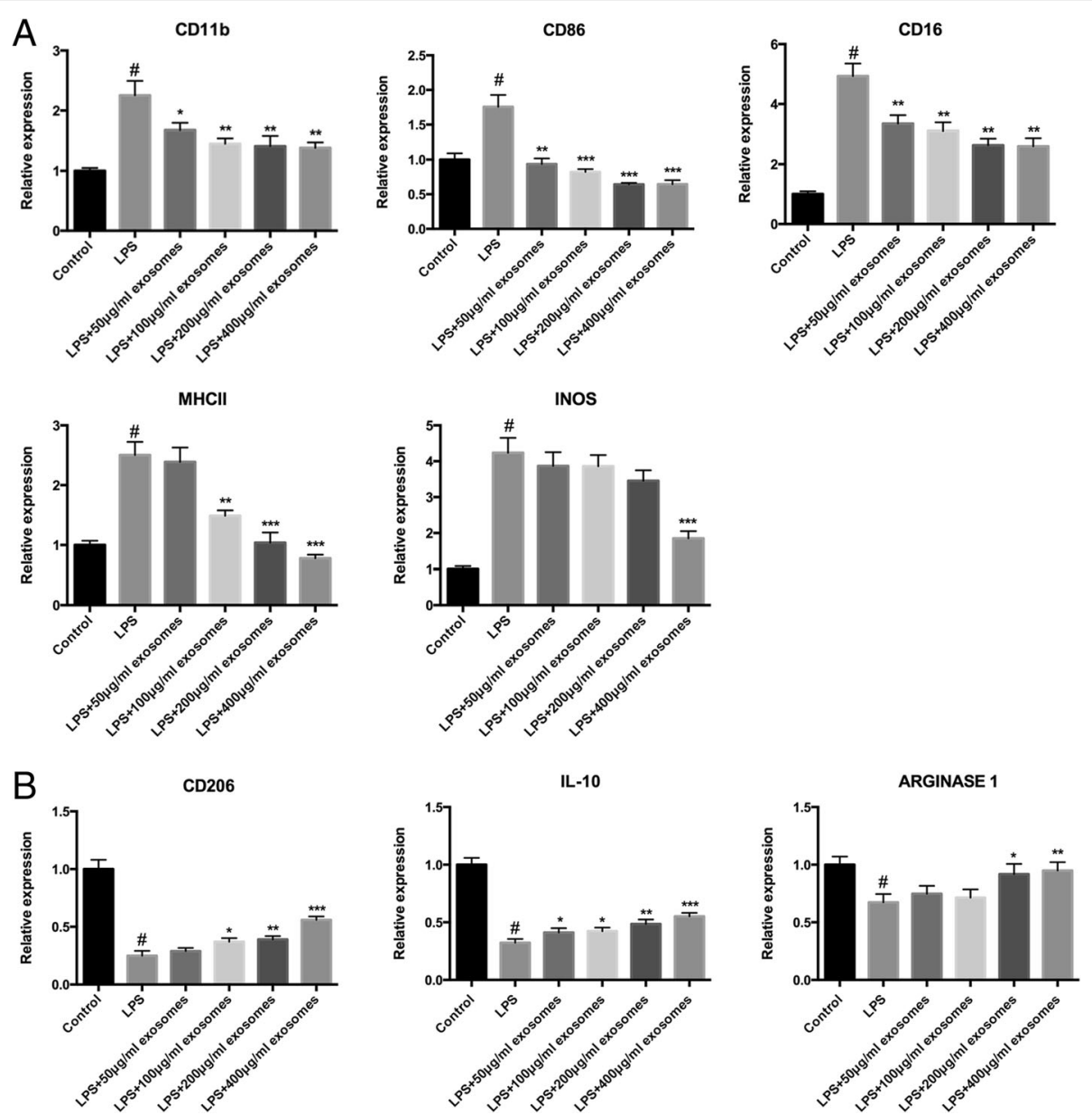

Fig. 5 Detection of mRNA expression of M1 and M2 phenotypic markers of microglia. a M1-associated markers CD11b, CD86, CD16, MHC II, and iNOS were significantly decreased after exosome treatment compared with the single LPS stimulation group. b M2-associated markers CD206, IL10, and ARGINASE 1 were significantly increased after exosome treatment compared to the single LPS stimulation group. ${ }^{\#} P<0.05$ compared with non-activated microglia cells. ${ }^{*} P<0.05,{ }^{* * *} P<0.01,{ }^{* * *} P<0.001$ compared with LPS-only treated cells. IL-10 interleukin-10, LPS lipopolysaccharide

functions, which is in line with our results [33]. Microglia have been reported as the most important resident immune cells in the central nervous system [34]. After TBI, microglia were soon activated to release pro-inflammatory factors, for example, IL- 6 and TNF- $\alpha$, which may in turn exacerbate brain damage. The reduced release of these inflammatory factors by SHED treatment may be beneficial in TBI by inhibiting neuroinflammation. Nitrite is the end product of NO, which plays neurotoxic roles after TBI. Therefore, the decreased nitrite concentration in the SHED co-culture group may also account for the protective effects found in TBI.

The present study demonstrated that after co-culturing SHED with microglia for $48 \mathrm{~h}$ in the transwell system, the concentration of nitrite and inflammatory factors IL- 6 and TNF- $\alpha$ all decreased compared with the single activated microglia group. As is reported previously, MSCs could secrete anti-inflammatory growth factors $[35,36]$. While increasing data suggest that exosomes derived from mesenchymal stem cells was an also an important player in repairing tissue damage [37]. Therefore, we hypothesize that exosomes produced by the co-cultured SHED (SHEDEx) may contribute the decreased secretion of inflammatory factors by microglia. To clarity if and to what extent SHED-Ex influence the co-cultured microglia, we designed the following experiments. We found that purified SHEDEx was alone able to alter the polarization of microglia, and inhibit the inflammatory effects of M1 microglia. Therefore, we concluded that SHED-Ex, independent of the anti-inflammatory growth factors, could reduce inflammation, to an even larger extent than the reduction effect that was found in co-culture systems. It is reported that exosomes transport RNAs, proteins, and lipids to the targeted cells to reprogram cell behaviors [38]. Among them, noncoding RNAs, such as miRNAs or LncRNAs, have been reported to play crucial roles. Therefore, further studies are warranted to focus on specific noncoding RNAs that dominate the process. Once the specific molecule is 


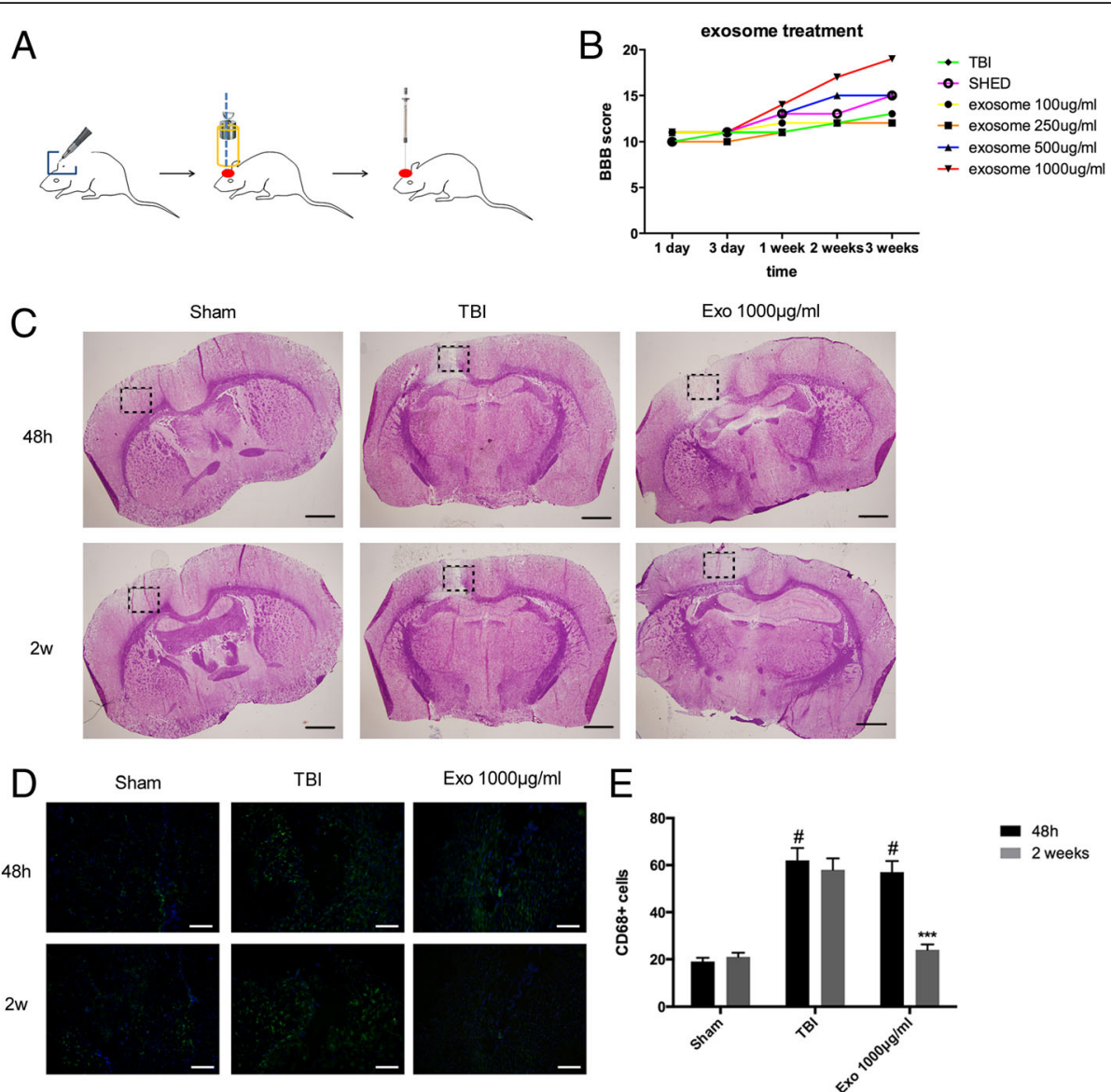

Fig. 6 SHED-Ex promotes functional motor recovery after TBI. a Establishment of the TBI model in rats. b Motor assessment. The Basso, Beattie, and Bresnahan (BBB) scores for each group from 1 to 21 days after TBI. $\mathbf{c}$ Lesion reconstruction at $48 \mathrm{~h}$ and 2 weeks post-transplant. Scale bar = $50 \mu \mathrm{m}$ (d) CD68 staining for activated microglia at $48 \mathrm{~h}$ and 2 weeks post-transplant. Scale bar $=50 \mu \mathrm{m}$. (e) Treatment with SHED-Ex significantly reduces the number of CD68+ microglia in the brains of rats after TBI. ${ }^{\sharp} P<0.05$ compared with sham group. ${ }^{* * *} P<0.001$ compared with TBI group. SHED-Ex stem cells from human exfoliated deciduous teeth-originated exosomes, TBI traumatic brain injury

revealed, the therapeutic efficiency will be enhanced by selective manipulation of the expression.

It has been demonstrated that microglia-mediated neuroinflammation plays a critical role in secondary brain injury in TBI [13]. In the present study, SHED-Ex could significantly reduce the pro-inflammatory microglia M1 phenotype cell markers; more importantly, it could do so in a dose-dependent manner. To further certify the role of SHED-Ex on microglial polarization, the mRNA levels of microglia M1/M2 phenotype markers were detected. As we hypothesized, after incubation with SHED-Ex for $48 \mathrm{~h}$, a group of microglia was polarized from the pro-inflammatory phenotype into the anti-inflammatory phenotype, which led to a welcoming restoration after neuroinflammation. In this case, SHEDEx were further investigated to detect the neuroprotective roles they may have played when administered to rats with TBI.

In the current study, $500 \mu \mathrm{g} / \mathrm{ml} \mathrm{SHED-Ex} \mathrm{injected} \mathrm{into}$ rat brain rescued the cortical damage and improved the motor deficits resulting from TBI in rats. Actually, it was almost equivalent to the effect that occurred with a $10^{5}$ / $3 \mu \mathrm{l}$ SHED treatment for TBI, while $1000 \mu \mathrm{g} / \mathrm{ml}$ exosomes provided a better functional recovery. Therefore, the dose-response efficacy was determined. However, whether a higher dose could provide better efficacy is unknown. Additionally, CD68+ microglia were used to identify activated M1 microglia after TBI. Compared with the TBI group, $1000 \mu \mathrm{g} / \mathrm{ml}$ SHED-Ex could significantly suppress the CD68+ microglia, which suggested the potential therapeutic mechanisms of SHED-Ex transplantations. In addition, we cannot ignore the possibility of how SHED-Ex may also act, such as the benefits of direct or indirect neurovascular regeneration. Future investigations are therefore planned to determine whether neurovascular regeneration could also be manipulated by SHED-Ex.

We combined the above data to explain the therapeutic mechanism of SHED-Ex. At the very beginning of TBI, microglia become more polarized towards M1 activation 


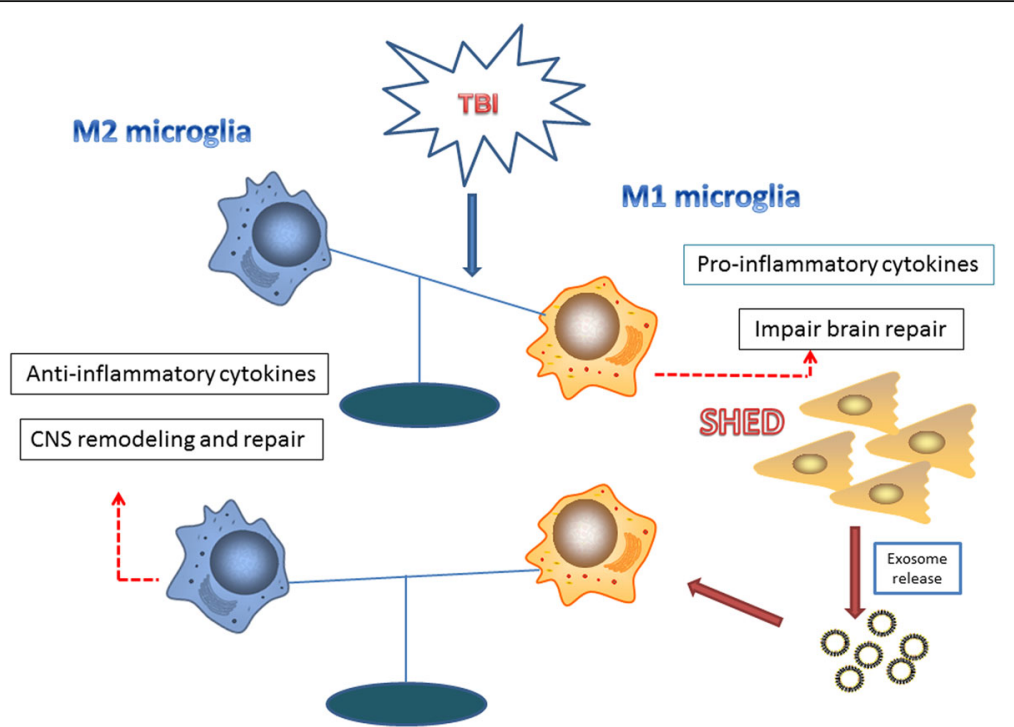

Fig. 7 Schematic of SHED-Ex repair of impaired CNS: SHED-Ex shifts microglia M1/M2 polarization. SHED-Ex contact with activated microglia to promote robust M2 polarization. As a result, anti-inflammatory cytokines were released to repair. CNS central nervous system, SHED-Ex stem cells from human exfoliated deciduous teeth-originated exosomes, TBI traumatic brain injury

states over M2. By releasing pro-inflammatory cytokines as well as free radicals, M1 microglia-mediated chronic neuroinflammation exacerbated neurological impairments. In contrast, when SHED-Ex were added, M2 microglia were robustly activated, and thus the neuroinflammation was suppressed by anti-inflammatory cytokines. Long-term functional recovery and reduced neurodegeneration are therefore developed (Fig. 7).

\section{Conclusions}

The current study demonstrated for the first time that the administration of SHED-Ex improves rat motor functional recovery and reduces neuroinflammation after TBI by shifting microglia M1/M2 polarization. This result may provide a novel therapeutic approach for the management of TBI and probably other neurological disorders.

\section{Abbreviations}

BBB: Basso, Beattie, and Bresnahan; DFPCs: Dental follicle progenitor cells; DMEM: Dulbecco's modified Eagle's medium; DPSCs: Dental pulp stem cells; ELISA: Enzyme-linked immunosorbent assay; FBS: Fetal bovine serum; IL6: Interleukin-6; LPS: Lipopolysaccharide; MSCs: Mesenchymal stem cells; NSCs: Neural stem cells; PDLSCs: Periodontal ligament stem cells; SCAP: Stem cells from apical papilla; SHED: Stem cells from human exfoliated deciduous teeth; SHED-Ex: Stem cells from human exfoliated deciduous teethoriginated exosomes; TBI: Traumatic brain injury; TNF-a: Tumor necrosis factor alpha

\section{Acknowledgements}

We are very grateful to Prof. LV Hai-Xia (Xi'an Jiaotong University School of Medicine) for kindly helping us with animal models.

\section{Funding}

The current work was supported by Shaanxi Provincial Science and Technology Innovation Project co-ordination of Resources Oriented Industries of Key Technologies Project No. 2011KTCL03-24.

\section{Availability of data and materials}

All data generated or analyzed during this study are included in this published article.

\section{Author contributions}

$Y L, Y Y, J R$, and AL contributed to the conception and design of the study, $Y L$, FX, FC and AL did the job of acquisition, analysis, and interpretation of data, and $\mathrm{YL}$ drafted the manuscript. All authors approved the final manuscript.

\section{Ethics approval}

The study has been approved by the ethics board of College of Stomatology, Xi'an Jiaotong University. All experiments conformed to the guidelines issued by the committee on animal research of College of Stomatology, Xi'an Jiaotong University.

Consent for publication

Not applicable

\section{Competing interests}

The authors declare that they have no competing interests.

\section{Publisher's Note}

Springer Nature remains neutral with regard to jurisdictional claims in published maps and institutional affiliations.

\footnotetext{
Author details

${ }^{1}$ Key Laboratory of Shaanxi Province for Craniofacial Precision Medicine Research, Xi'an, Shaanxi, China. ${ }^{2}$ Department of Periodontology, College of Stomatology, Xi'an Jiaotong University, Xi Wu Road No.98, Xi'an, Shaanxi 710004, China. ${ }^{3}$ The Key Laboratory of Biomedical Information Engineering of Ministry of Education, Xi'an Jiaotong University School of Life Science and Technology, Xi'an, China. ${ }^{4}$ Bioinspired Engineering and Biomechanics Center (BEBC), Xi'an Jiaotong University, Xi'an, China. ${ }^{5}$ Department of Periodontology, School of Stomatology, Fourth Military Medical University, Xi'an, People's Republic of China.
} 
Received: 7 May 2017 Revised: 11 August 2017

Accepted: 14 August 2017 Published online: 29 September 2017

\section{References}

1. Vakhrushev IV, Antonov EN, Popova AV, et al. Design of tissue engineering implants for bone tissue regeneration of the basis of new generation polylactoglycolide scaffolds and multipotent mesenchymal stem cells from human exfoliated deciduous teeth (SHED cells). Bull Exp Biol Med. 2012; 153(1):143-7.

2. Rosa V, Zhang Z, Grande RHM, Nör JE. Dental pulp tissue engineering in full-length human root canals. J Dent Res. 2013;92(11):970-5.

3. Miura M, Gronthos S, Zhao M, et al. SHED: Stem cells from human exfoliated deciduous teeth. Proc Natl Acad Sci U S A. 2003:100(10):5807-12.

4. Annibali S, Cristalli MP, Tonoli F, Polimeni A. Stem cells derived from human exfoliated deciduous teeth: a narrative synthesis of literature. Eur Rev Med Pharmacol Sci. 2014;18(19):2863-81.

5. Nishino $Y$, Yamada $Y$, Ebisawa K, et al. Stem cells from human exfoliated deciduous teeth (SHED) enhance wound healing and the possibility of novel cell therapy. Cytotherapy. 2011;13(5):598-605.

6. Kanafi MM, Rajeshwari YB, Gupta S, et al. Transplantation of islet-like cell clusters derived from human dental pulp stem cells restores normoglycemia in diabetic mice. Cytotherapy. 2013;15(10):1228-36.

7. Ma L, Makino Y, Yamaza H, et al. Cryopreserved dental pulp tissues of exfoliated deciduous teeth is a feasible stem cell resource for regenerative medicine. PLoS One. 2012;7(12):e51777.

8. Ueda M, Nishino Y. Cell-based cytokine therapy for skin rejuvenation. J Craniofac Surg. 2010;21(6):1861-6.

9. Daltoé FP, Mendonça PP, Mantesso A, Deboni MC. Can SHED or DPSCs be used to repair/regenerate non-dental tissues? A systematic review of in vivo studies. Braz Oral Res. 2014;28(1):1-7.

10. Greve MW, Zink BJ. Pathophysiology of traumatic brain injury. Mt Sinai J Med. 2009;76(2):97-104.

11. Xiong Y, Zhang Y, Mahmood A, Chopp M. Investigational agents for treatment of traumatic brain injury. Expert Opin Investig Drugs. 2015;24(6):743-60.

12. Brites D, Fernandes A. Neuroinflammation and depression: microglia activation, extracellular microvesicles and microRNA dysregulation. Front Cell Neurosci. 2015:9:476.

13. Kutteh WH, Kantele A, Moldoveanu Z, Crowley-Nowick PA, Mestecky J. Neuroinflammation after traumatic brain injury: opportunities for therapeutic intervention. Brain Behav Immun. 2012;26(8):1191-201.

14. Zomer A, Vendrig T, Hopmans ES, Eijndhoven M, Middeldorp JM, Pegtel DM. Exosomes. Commun Integr Biol. 2010;3(5):447-50.

15. Arslan F, Lai RC, Smeets MB, et al. Mesenchymal stem cell-derived exosomes increase ATP levels, decrease oxidative stress and activate PI3KJAkt pathway to enhance myocardial viability and prevent adverse remodeling after myocardial ischemia/reperfusion injury. Stem Cell Res. 2013;10(3):301-12.

16. Nakamura Y, Miyaki S, Ishitobi H, et al. Mesenchymal-stem-cell-derived exosomes accelerate skeletal muscle regeneration. Febs Letters. 2015; 589(11):1257-65.

17. Dorronsoro A. Regenerating the injured kidney with human umbilical cord mesenchymal stem cell-derived exosomes. Stem Cell Res Ther. 2013;4(2):2239-46.

18. Marote A, Teixeira FG, Mendes-Pinheiro B, Salgado AJ. MSCs-derived exosomes: cell-secreted nanovesicles with regenerative potential. Front Pharmacol. 2016;7:231.

19. Rani S, Ryan AE, Griffin MD, Ritter T. Mesenchymal stem cell-derived extracellular vesicles: toward cell-free therapeutic applications. Mol Ther 2015;23(5):812-23

20. Yin Z, Wang Q, Li Y, Wei H, Shi J, Li A. A novel method for banking stem cells from human exfoliated deciduous teeth: lentiviral TERT immortalization and phenotypical analysis. Stem Cell Res Ther. 2016;7:50.

21. Jose S, Tan SW, Tong CK, Vidyadaran S. Isolation and characterization of primary microglia from post-natal murine brain tissues: a comparison of two methods. Cell Biol Int. 2015;39(12):1355-63.

22. Gu J, Qian H, Shen L, et al. Gastric cancer exosomes trigger differentiation of umbilical cord derived mesenchymal stem cells to carcinoma-associated fibroblasts through TGF-B/Smad pathway. PLoS One. 2012;7(12):e52465.

23. Feeney DM, Boyeson MG, Linn RT, Murray HM, Dail WG. Responses to cortical injury: I. Methodology and local effects of contusions in the rat. Brain Res. 1981;211(1):67-77.

24. Basso DM, Beattie MS, Bresnahan JC. A sensitive and reliable locomotor rating scale for open field testing in rats. J Neurotrauma. 1995;12(1):1-21.
25. Yeh YC, Nitadori J, Kadota K, et al. Using frozen section to identify histological patterns in stage I lung adenocarcinoma of $\leq 3 \mathrm{~cm}$ : accuracy and interobserver agreement. Histopathology. 2015;66(7):922-38.

26. Shear DA, Tate MC, Archer DR, et al. Neural progenitor cell transplants promote long-term functional recovery after traumatic brain injury. Brain Res. 2004;1026(1):11-22.

27. Harting MT, Sloan LE, Jimenez F, Baumgartner J, Cox Jr CS. Subacute neural stem cell therapy for traumatic brain injury. J Surg Res. 2009;153(2):188-94.

28. Mahmood A, Lu D, Qu C, Goussev A, Chopp M. Human marrow stromal cell treatment provides long-lasting benefit after traumatic brain injury in rats. Neurosurgery. 2005;57(5):1026-31.

29. Guan J, Zhu Z, Zhao RC, et al. Transplantation of human mesenchymal stem cells loaded on collagen scaffolds for the treatment of traumatic brain injury in rats. Biomaterials. 2013;34(24):5937-46.

30. Tajiri N, Acosta SA, Shahaduzzaman M, et al. Intravenous transplants of human adipose-derived stem cell protect the brain from traumatic brain injury-induced neurodegeneration and motor and cognitive impairments: cell graft biodistribution and soluble factors in young and aged rats. $J$ Neurosci. 2014;34(1):313-26.

31. Huang GT, Gronthos S, Shi S. Mesenchymal stem cells derived from dental tissues vs. those from other sources: their biology and role in regenerative medicine. J Dent Res. 2009:88(9):792-806.

32. Tsai CL, Chuang PC, Kuo HK, Chen YH, Su WH, Wu PC. Differentiation of stem cells from human exfoliated deciduous teeth toward a phenotype of corneal epithelium in vitro. Cornea. 2015;34(11):1471-7.

33. Zhang $R$, Liu $Y$, Yan $K$, et al. Anti-inflammatory and immunomodulatory mechanisms of mesenchymal stem cell transplantation in experimental traumatic brain injury. J Neuroinflammation. 2013;10:106.

34. Harry GJ. Neuroinflammation: a need to understand microglia as resident cells of the developing brain. NeuroToxicology. 2012;33(3):558-9.

35. Buul GMV, Villafuertes E, Bos PK, et al. Mesenchymal stem cells secrete factors that inhibit inflammatory processes in short-term osteoarthritic synovium and cartilage explant culture. Osteoarthr Cartil. 2012;20(10):1186-96.

36. Oh JY, Kim MK, Shin MS, et al. The anti-inflammatory and anti-angiogenic role of mesenchymal stem cells in corneal wound healing following chemical injury. Stem Cells. 2008;26(4):1047-55.

37. Turturici G, Tinnirello R, Sconzo G, Geraci F. Extracellular membrane vesicles as a mechanism of cell-to-cell communication: advantages and disadvantages. Am J Physiol Cell Physiol. 2014;306(7):621-33.

38. Ferguson S, Nguyen J. Exosomes as therapeutics: The implications of molecular composition and exosomal heterogeneity. J Control Release. 2016:228:179-90.

\section{Submit your next manuscript to BioMed Central and we will help you at every step:}

- We accept pre-submission inquiries

- Our selector tool helps you to find the most relevant journal

- We provide round the clock customer support

- Convenient online submission

- Thorough peer review

- Inclusion in PubMed and all major indexing services

- Maximum visibility for your research

Submit your manuscript at www.biomedcentral.com/submit 